\title{
New Recommendations for Cardiopulmonary Resuscitation and Emergency Cardiovascular Care
}

To the Editor,

The year has begun with several news in Cardiopulmonary Resuscitation (CPR), and at the end of 2010 new guidelines for CPR and Emergency Cardiovascular Care (ECC) were published ${ }^{1}$. The matter is of great importance for the entire medical community, especially for anesthesiologists who are often involved in resuscitation. New priorities are given to cardiac arrest. On the other hand, approaches adopted long ago now have less importance. It is the evidence-based medicine. The catchwords of the new publication are: "high-quality chest compressions" and "simplification". The terminology "the mean of 100 compressions per minute" has been substituted by "at least 100 compressions per minute". The procedure "look, listen, and feel" related to assessment of the airways in previous guidelines ${ }^{2}$ was excluded. Atropine is no longer indicated for treating pulseless electrical activity (PEA) and asystole. Post-resuscitation care has been included as part of the survival chain. Greater importance has also been given to capnography, and the precordial thump is once again recommended in special situations.

According to the new guidelines high-quality chest compressions on adult patients are defined as those with a minimum frequency of 100 per minute and a minimum depth of $5 \mathrm{~cm}$ with complete chest recoil after each compression, reducing the no-flow time (for example, after defibrillation, to guarantee the airways or to check the pulse). Excessive ventilation should be avoided. The pattern of two ventilations for 30 chest compressions, or 8 to 10 ventilations per minute, asynchronic with chest compressions, if the airways have been secured with any device (tracheal tube of laryngeal mask, for example) is maintained. Different from what had been practiced, currently high-quality chest compressions are recommended as the first step on the cardiac arrest algorithm followed by maintenance of airway patency, and ventilation - C (chest compressions), A (airway), B (breathing), and no longer the $A, B, C$ sequence.

Lay rescuers or those less trained should only perform chest compressions (hands only) making fast decisions after a brief evaluation of the responsiveness of the victim and whether he/ she is breathing normally or not at all. Literature reports that early onset of chest compressions improves the prognosis of cardiac arrest. The lay rescuers should maintain chest compressions until a defibrillator or a trained paramedic is available.

The health care professional should observe ventilation while also checks if the patient is responding (consciously or not), and then start immediately 30 high-quality chest compressions followed by cleaning the airway and performing two ventilations. If the professional is not alone these actions can be performed simultaneously remembering that chest compressions are priority. In case of ventricular fibrillation or pulseless ventricular tachycardia the defibrillator should be used as soon as possible. In patients with witnessed collapse, while the automatic defibrillator is being connected, chest compressions should be maintained and soon after the shock they should be reinstated. When out-of-hospital cardiac arrest is not witnessed, consider chest compressions for approximately one and half to three minutes before any attempts to defibrillate. The patient should be shocked up to three minutes after collapsing, when indicated.

On the 2005 edition $^{2}$, there were no recommendations on precordial thump. This technique has been included in the new edition as an alternative for witnessed unstable ventricular tachycardia in monitored patient when a defibrillator is not readily available. Again, the publication emphasizes that the precordial thump should not delay CPR neither the shock.

More attention has been given to capnography. In the past it was subtly recommended to confirm positioning of the endotracheal tube and for being useful as an indication of the cardiac output generated during chest compressions; currently it is strongly recommended for the same purposes including the detection of increased blood flow when spontaneous circulation returns.

Drug protocols have also been modified. The routine use of atropine in cases of asystole or PEA was excluded from the algorithm because evidence is not solid to indicate its benefit. Furthermore, adenosine can be used in cases of regular widecomplex monomorphic tachycardia as therapeutic and diagnostic tool, and not only in cases of supraventricular tachycardia.

Finally, organized post-resuscitation care such as implementation of an encompassing, structured, integrated, and multidisciplinary system of care is emphasized; i.e., continuous evaluation and hemodynamic, respiratory, and neurologic support including percutaneous coronary interventions, when indicated, as well as hypothermia after all types of cardiac arrest. Maintaining the arterial oxygen saturation between $94 \%$ and $99 \%$ avoiding postresuscitation hyperoxia is considered ideal.

Given the news on such an important matter in our field, we emphasize the need for updating and continuing education. The new guidelines can be obtained for free on the website of the American Heart Association ${ }^{1}$. The highlights of the new guidelines, also for free, have been published in several languages including Portuguese ${ }^{3}$. The medical community should be attentive and trained mastering an old subject that was renewed for 2011.

Dra. Camila Sampaio Chiarantano ME of CET/SBA of Departamento de Anestesiologia Faculdade de Medicina de Botucatu

Dr. Paulo do Nascimento Junior Associate Professor of the Departamento de Anestesiologia Faculdade de Medicina de Botucatu 


\section{REFERÊNCIAS / REFERENCES}

01. 2010 American Heart Association guidelines for cardiopulmonary resuscitation and emergency cardiovascular care science. Circulation, 2010;122(18 suppl 3):S639-S946. Disponível em: http://www.circ.ahajournals.org/content/vol122/18_suppl_3/>

02. 2005 American Heart Association guidelines for cardiopulmonary resuscitation and emergency cardiovascular care. Circulation, 2005;112(24 suppl):iv1-iv211.

03. Destaques das diretrizes da American Heart Association 2010 para RCP e ACE. Disponível em: http://guidelines.ecc.org/guidelines-highlights.html 